\section{Percepção de saúde e fatores associados em adultos: inquérito populacional em Rio Branco, Acre, Brasil, 2007-2008}

\author{
Self-rated health and associated factors in adults: \\ a population survey in Rio Branco, Acre State, \\ Brazil, 2007-2008
}

${ }^{1}$ Centro de Ciências da Saúde e do Desporto, Universidade Federal do Acre Rio Branco, Brasil.

2 Escola Nacional de Saúde Pública Sergio Arouca, Fundação Oswaldo Cruz Rio de Janeiro, Brasil.

Correspondência P. C. L. Bezerra Centro de Ciências da Saúde e do Desporto, Universidade Federal do Acre. Campus Universitário, Centro de Ciências da Saúde e do Desporto, Rodovia BR 364 , Km 04, Rio Branco, AC 69915-900, Brasil. polyana_caroline@yahoo. com. br

\section{Abstract}

The subjective concept of health included in the self-rated health process allows identifying factors associated with good self-rated health in populations. This cross-sectional population-based study in 2007-2008 focused on self-rated health and associated factors among adults $(n=1,516)$ in Rio Branco, Acre State, Brazil. The multivariate analysis used Poisson regression, with fair/ poor self-rated health as the dependent variable. Differences in gender, age group, level of schooling, and income negatively affected individuals self-rated health. After adjusting for age, gender, and self-reported illness, the estimated risks for fair/poor health were higher for the unemployed, obese, and those with some self-reported illness. The results suggest that differences in living conditions for adults in Rio Branco are associated with precarious self-rated health.

Health Status; Diagnosis of Health Situation; Health Profile; Health Surveys
Polyana Caroline de Lima Bezerra 1 Simone Perufo Opitz 1 Rosalina Jorge Koifman ${ }^{2}$ Pascoal Torres Muniz 1

\section{Introdução}

A autoavaliação de saúde é cada vez mais investigada e retrata o estado de saúde da população, seja positivo, seja negativo, considerando a ótica pessoal do indivíduo. Entende-se que o conceito subjetivo de saúde automaticamente incluso nesse processo possibilita investigar quais são os fatores associados à percepção de vida saudável da população em estudo.

A percepção de saúde é uma avaliação global de saúde com base em uma análise dos aspectos objetivos e subjetivos de cada indivíduo. Neri 1 relata que a subjetividade refere-se ao modo como as pessoas se sentem, como julgam os componentes objetivos como fatores qualitativos de suas vidas. Quando o indivíduo avalia o seu estado de saúde, não o faz de forma circunstancial 2, mas, sim, como referência à análise de Canguilhem ${ }^{3}$ da saúde, como expressão dos "modos de andar a vida".

A percepção precária da própria saúde pode ser vista como resultado de sentimentos provocados pelo mal-estar, dor ou desconforto, em interação com os fatores sociais, culturais, psicológicos e ambientais que modificam a maneira como a vida da pessoa é afetada pelo problema experimentado. Dessa forma, essa percepção deve ser analisada sob uma ótica multidimensional, levando em consideração os diferentes entendimentos individuais da saúde, diante do contexto cultural e psicossocial existente. Essa 
autoavaliação repercute na qualidade de vida do indivíduo ${ }^{4}$.

A autoavaliação é uma medida de saúde validada com dados de indicadores clínicos de morbidade e de mortalidade, além de ser um bom marcador das diferenças nos subgrupos populacionais 4 . Diversos estudos recentes têm demonstrado que a autopercepção de saúde é um preditor de mortalidade 4,5,6

Por considerar a importância resultante desta investigação e a fim de auxiliar na discussão do tema como instrumento de qualidade de saúde das populações, a autoavaliação de saúde tem sido pesquisada em diversos estudos populacionais internacionais, procurando analisar o efeito do estado socioeconômico, entre outros fatores, na saúde das pessoas. Os resultados desses estudos demonstram que as mulheres, pessoas negras, de maior idade e baixa escolaridade referem mais negativamente seu estado de saúde 4,5,6,7,8.

No Brasil, os estudos de base populacional que se propuseram a investigar as desigualdades sociais em saúde por meio da autopercepção desta revelaram forte associação entre condições socioeconômicas desfavoráveis e características demográficas específicas com a pior percepção do estado de saúde 9,10,11,12,13. Outras pesquisas nacionais de saúde sugerem que diferenças entre gênero, faixas etárias e nível de instrução afetam a percepção de saúde dos indivíduos 9,14,15,16,17, corroborando resultados da literatura internacional $4,7,8$.

Este estudo, estimou a prevalência da autopercepção relativa ao estado geral de saúde da população adulta residente no Município de Rio Branco, Estado do Acre. Em adição, procurou avaliar as relações de associação da percepção "insatisfatória" de saúde com base na distribuição de características socioeconômicas, demográficas, de hábitos de vida e de situação de saúde, como índice de massa corporal e número de morbidades autorreferidas.

\section{Métodos}

Trata-se de um estudo transversal de base populacional, constituído por indivíduos com 18 anos ou mais, residentes do Município de Rio Branco, entre 2007 e 2008. Este trabalho é parte integrante da pesquisa Saúde e Nutrição em Crianças e Adultos no Município de Rio Branco, Acre, realizada pela Universidade Federal do Acre em parceria com a Secretaria de Vigilância em Saúde do Ministério da Saúde, Secretaria Estadual de Saúde do Acre e Secretaria Municipal de Saúde do Município de Rio Branco.
O desenho amostral empregado foi uma amostra por conglomerados com duas etapas de sorteio. A unidade amostral primária foi o setor censitário definido pelo Instituto Brasileiro de Geografia e Estatística (IBGE; http://www.ibge. gov.br). Dentre os 2.450 setores censitários, foram sorteados 35 (31 urbanos e 4 rurais) e, em cada um, foram sorteados 25 domicílios, tendo como base o mapa elaborado pela Pesquisa Nacional por Amostra de Domicílios (PNAD) de 2006.

Entrevistaram-se todos os moradores adultos residentes em cada domicílio sorteado. Nos casos de ausência de algum componente no momento da primeira visita, foram agendados retornos aos domicílios para contemplar todos os moradores destes, o que fez com que a amostra tivesse uma estrutura por sexo e idade semelhante à da população do município. O critério de inclusão no estudo foi a anuência de todos adultos à participação da pesquisa. Excluindo as perdas e recusas, foram totalizados 1.516 participantes (850 domicílios).

O desenho e amostragem, assim como outros aspectos metodológicos deste estudo, foram descritos mais detalhadamente em Lino et al. 18 .

Para a coleta de dados, elaborou-se um questionário, com questões fechadas, semiabertas e abertas, constituído de módulos organizados por conjuntos temáticos. Esse instrumento baseouse em dois inquéritos: o Inquérito Domiciliar sobre Comportamentos de Risco e Morbidade Referida de Doenças e Agravos não Transmissíveis: Brasil, 15 capitais e Distrito Federal, 2002-2003 12 e o Vigilância de Fatores de Risco e Proteção para Doenças Crônicas por Inquérito Telefônico (VIGITEL 2006) 19. Nem todos os blocos/módulos foram aplicados a todos os entrevistados, pois alguns temas eram específicos para determinadas condições individuais.

O questionário contemplou as seguintes variáveis de interesse deste estudo: demográficas (sexo, grupo etário, raça/cor), socioeconômicas (estado conjugal, escolaridade, situação ocupacional, renda familiar), além de aspectos relacionados a hábitos de vida (prática de atividade física, tabagismo, consumo de bebida alcoólica, hábito alimentar saudável) e à saúde autorreferida (percepção de saúde, morbidades autorreferidas). Contemplou, ainda, medida de peso e altura, permitindo o cálculo do índice de massa corporal (IMC).

A autopercepção de saúde foi caracterizada em quatro níveis: "excelente", "boa”, "regular" e "ruim". O estrato "não sabe” foi considerado como perda $(\mathrm{n}=2)$. Para a análise da percepção insatisfatória de saúde, utilizada como desfecho, foi criada uma variável dicotômica construída a partir da variável "percepção de saúde”, na qual 
se classificaram as seguintes categorias: percepção de saúde insatisfatória, unindo os estratos: regular e ruim, e percepção satisfatória: excelente e boa.

A faixa etária foi estratificada em 18-29, 3039, 40-49, 50-59 e 60 anos ou mais. A cor da pele/ etnia autodeclarada foi dicotomizada em preta e/ou parda (sim/não). A variável escolaridade foi dividida em quatro categorias: 0; 1 a 7; 8 a 10; 11 ou mais anos de estudo. As informações sobre atividades ocupacionais foram agrupadas em com e sem ocupação. O estado conjugal foi dividido em duas categorias: com e sem companheiro. Foram definidas quatro categorias de renda familiar: 0 ; $<1$ a $<3$; 3 a $<5$; 5 ou mais salários mínimos, sendo considerado o salário mínimo em vigor ( $\mathrm{R} \$ 465,00)$.

Nas variáveis de hábitos de vida, o tabagismo foi representado pela variável dicotômica, sendo considerado como fumantes os indivíduos que assim se denominavam no momento da entrevista e aqueles que já tinham fumado anteriormente. O etilismo foi classificado segundo afirmação ou negação do entrevistado ao questionamento de consumo de bebida alcoólica.

Para a prática de atividade física, foi criada uma variável dicotômica com referência à classificação da Organização Mundial da Saúde (OMS) 20 sobre prática de atividade física de lazer e de atividade física ocupacional. Considerou-se prática de atividade física ocupacional quando o indivíduo respondeu "sim" a pelo menos uma das questões: "No seu trabalho, o(a) sr.(a) anda bastante a pé?"; "No seu trabalho, o(a) sr.(a) carrega peso ou faz outra atividade pesada?"; " $O(A)$ sr.(a) costuma ir a pé ou de bicicleta de casa para o trabalho?"; "O(A) sr. (a) costuma ter ajuda para fazer faxina?"; "Quem fica com a parte mais pesada da faxina?”. Já a prática de atividade física de lazer foi classificada segundo afirmação do entrevistado ao responder se faz algum tipo de exercício físico. Portanto, a variável dicotômica "prática de atividade física" foi criada considerando as respostas das duas variáveis supracitadas.

Para avaliar o hábito alimentar, foi criado um “índice de alimentação saudável” baseando-se em Figueiredo et al. ${ }^{21}$. As variáveis referentes à alimentação foram coletadas por meio de escala de frequência de consumo alimentar (1 a 2 dias por semana; 3 a 4 dias por semana; 5 a 6 dias por semana; todos os dias; quase nunca ou nunca), que foram subsequentemente dicotomizadas: consumo usual ("todos os dias"/" de 1 a 6 dias por semana”) e consumo não usual ("quase nunca" / "nunca”). As variáveis foram classificadas como marcadoras de consumo alimentar saudável (peixe, feijão, frutas, legume ou vegetal cozido, gordura vegetal, salada crua) e marcadoras de consumo alimentar menos saudável (leite integral, carne vermelha com gordura, frango com pele, refrigerante). Estas variáveis foram dicotomizadas em consumo usual ( $\operatorname{sim/não)~e~codifica-~}$ das como "0" para sim e "1" para não, nas variáveis marcadoras de consumo alimentar saudável; “0” para não e " 1 ” para sim, nas variáveis marcadoras de consumo alimentar menos saudável. A soma total dessas variáveis é igual a 10. Definiu-se o valor "3" como ponto de corte, sendo a pontuação de 0 a 3 considerada hábito alimentar mais saudável e de 4 a 10, hábito alimentar menos saudável. Construiu-se, assim, a variável dicotômica alimentação saudável (sim/não).

O IMC foi categorizado conforme preconizado pela OMS 22: baixo peso: < 18,5; eutrófico: 18,5-24,9; sobrepeso: 25-29,9; obesidade: $\geq 30$.

A variável morbidade autorreferida foi construída valendo-se da resposta a uma listagem de 18 morbidades e pôde ser analisada de duas formas: como variável categórica: nenhuma, 1-3; 4-6; 7-9 e de 10 ou mais; como variável dicotômica ( $\operatorname{sim} /$ não).

Os dados do inquérito populacional foram duplamente digitados e validados, utilizandose o software Epi Info 6.04 (Centers for Disease Control and Prevention, Atlanta, Estados Unidos). Para a análise estatística, utilizou-se o software Stata/SE 10.0 (Stata Corp., College Station, Estados Unidos), módulo survey, levando-se em conta o desenho da amostra e as ponderações necessárias. Foram estimadas as prevalências e as razões de prevalência bruta e ajustadas, utilizando regressão de Poisson, com ajuste robusto da variância, níveis de significância de 0,05 e intervalo de $95 \%$ de confiança. Para estimar as razões de prevalência ajustadas, inicialmente foi realizada análise bivariada, que verificou associação de percepção insatisfatória do estado de saúde com variáveis socioeconômicas, estilo de vida e situação de saúde. As associações que apresentaram valor de $\mathrm{p}<0,20$ foram incluídas no modelo multivariado. Mantiveram-se no modelo final: idade, sexo e morbidade autorreferida. Nas análises multivariadas que incluíram as variáveis do modelo final, estas foram ajustadas apenas pelas demais variáveis intervenientes. Por exemplo, na análise multivariada de idade, esta foi ajustada por sexo e morbidade autorreferida, assim como as outras variáveis incluídas no modelo.

Foram seguidas as recomendações da Resolução $n^{o}$. 196/96, do Conselho Nacional de Saúde do Ministério da Saúde, bem como a solicitação de autorização dos sujeitos-alvo deste estudo por meio do Termo de Consentimento Livre e Esclarecido. O projeto de pesquisa foi aprovado pelo Comitê de Ética em Pesquisa 
da Universidade Federal do Acre (protocolo 2307.001150/2007-22).

\section{Resultados}

A amostra total $(\mathrm{n}=1.516)$ foi composta por $55,9 \%$ de mulheres $(n=863)$ e $44,1 \%$ de homens ( $\mathrm{n}=653$ ). A média de idade na amostra como um todo foi de 38,8 anos (IC95\%: 37,1-40,5), sendo semelhantes as médias de idade das mulheres (39,0; IC95\%: 37,4-40,6) e dos homens (38,5; IC95\%: 36,4-40,7).

Ao serem analisadas as faixas etárias da população de Rio Branco, as mulheres apresentaram maiores percentuais em todos os estratos do que os homens, tendo oscilado de $52,9 \%$ nos indivíduos de 18 a 29 anos a 69,9\% naqueles com 40 a 49 anos.

A maioria da população $(84,2 \%)$ autodeclarou-se negra ou parda, com o percentual de $6,2 \%$ e $78 \%$ respectivamente. Apenas nove pessoas se autodeclararam de raça/cor amarela e nenhuma como indígena.
A média de renda familiar foi de $\mathrm{R} \$ 702,02$ (IC95\%: 525,23-878,81), porém as mulheres (R\$ 556,24; IC95\%: 395,91-716,56) apresentaram menores médias de renda familiar em relação aos homens (R\$ 941,84; IC95\%: 652,86-1.230,82). Quase a metade $(45,86 \%)$ da população analisada relatou não ter ocupação $(n=677)$. Quanto ao nível de instrução da amostra, $60,4 \%$ dos indivíduos tinham, pelo menos, o Ensino Fundamental completo (nove anos de estudo ou mais), e 6,7\% (IC95\%: 4,2-9,1) informaram ser analfabetos.

Na população investigada, 44,2\% (IC95\%: $39,9-55,2)$ das pessoas com 60 anos ou mais classificaram a própria saúde como regular; 18,6\% (IC95\%: 9,7-20,8), como ruim; 33,5\% (IC95\%: 24,5-38,8), como boa; e apenas 3,7\% (IC95\%: 2,0-9,0), como excelente. As mulheres tenderam a ter pior avaliação da própria saúde ao classificá-la como regular (40,3\%; IC95\%: $38,6-42,0)$ ou ruim $(9,7 \%$; IC95\%: 8,8-10,6) (Tabela 1). A prevalência de saúde avaliada como negativa (regular/ruim) aumentou significativamente com a idade, atingindo 62,9\% (IC95\%:

Tabela 1

Distribuição da frequência de percepção do estado de saúde em pessoas com 18 anos ou mais, segundo faixa etária e sexo. Inquérito populacional de Rio Branco, Acre, Brasil, 2007-2008 ( $n=1.514$ ).

\begin{tabular}{|c|c|c|c|c|c|}
\hline \multirow[t]{2}{*}{ Faixa etária (anos) } & \multirow[t]{2}{*}{ Tamanho da amostra } & \multicolumn{4}{|c|}{ Percepção de saúde (\%) } \\
\hline & & Excelente & Boa & Regular & Ruim \\
\hline \multicolumn{6}{|l|}{ Feminino } \\
\hline $18-29$ & 314 & 7,41 & 56,81 & 31,19 & 4,58 \\
\hline $30-39$ & 182 & 6,55 & 42,03 & 45,72 & 5,71 \\
\hline $40-49$ & 152 & 9,95 & 41,82 & 38,69 & 9,53 \\
\hline $50-59$ & 110 & 8,93 & 26,2 & 45,09 & 19,78 \\
\hline$\geq 60$ & 103 & 2,85 & 21,86 & 54,88 & 20,41 \\
\hline Total & 861 & 7,29 & 42,68 & 40,32 & 9,71 \\
\hline \multicolumn{6}{|l|}{ Masculino } \\
\hline $18-29$ & 269 & 18,52 & 56,00 & 22,94 & 2,55 \\
\hline $30-39$ & 133 & 12,62 & 52,14 & 29,47 & 5,79 \\
\hline $40-49$ & 97 & 16,58 & 45,78 & 26,77 & 10,87 \\
\hline $50-59$ & 77 & 14,13 & 32,59 & 51,93 & 13,5 \\
\hline$\geq 60$ & 77 & 4,56 & 46,73 & 32,08 & 16,63 \\
\hline Total & 653 & 14,59 & 49,6 & 29,72 & 6,09 \\
\hline \multicolumn{6}{|l|}{ Total } \\
\hline $18-29$ & 583 & 12,64 & 56,43 & 27,3 & 3,62 \\
\hline $30-39$ & 315 & 9,13 & 46,33 & 38,8 & 5,74 \\
\hline $40-49$ & 249 & 12,42 & 43,3 & 34,25 & 10,03 \\
\hline $50-59$ & 187 & 11,19 & 28,98 & 48,06 & 11,77 \\
\hline$\geq 60$ & 180 & 3,65 & 33,51 & 44,2 & 18,64 \\
\hline Total & 1.514 & 10,51 & 45,74 & 35,64 & 8,11 \\
\hline
\end{tabular}


$55,4-70,2)$ nos idosos e foi mais elevada no sexo feminino (Figura 1). Houve significância estatística $(\mathrm{p}<0,001)$ para todos os estratos.

$\mathrm{Na}$ Tabela 2, apresentam-se as prevalências e razões de prevalência ajustadas pelo modelo, que inclui: idade, sexo e morbidade autorreferida. A probabilidade de declaração de saúde regular/ruim cresce marcadamente nos idosos, sendo maior para as mulheres. A prevalência de saúde autoavaliada como "insatisfatória” (regular/ruim) foi maior nos idosos (62,9\%; IC95\%: 55,4-70,2), em comparação aos adultos entre 18 e 59 anos. Após o ajuste, os idosos apresentam prevalência $36 \%$ maior de autoclassificação da saúde como "insatisfatória" que os adultos. Encontramos associação entre percepção não boa de saúde e o sexo feminino $(\mathrm{RP}=1,38$; IC95\%: 1,01-1,88).

A prevalência de saúde avaliada como insatisfatória foi maior nos negros/pardos em relação aos não negros e pardos. A estimativa de risco ajustada foi $27 \%$ mais elevada para os negros/pardos. A razão de prevalência para a insatisfação com a saúde foi $19 \%$ menor nos indivíduos sem companheiros do que naqueles com companheiros (com significância estatística) (Tabela 2).

A porcentagem de pessoas que informaram que sua saúde era regular ou ruim foi de $56,6 \%$ (IC95\%: 45,9-67,7) para aqueles sem nenhuma escolaridade; 53,9\% (IC95\%: 50,7-57,1) no grupo de 1-7 anos de estudo; 35,2\% (IC95\%: 31,9-38,5) no grupo de 8-10 anos de estudo; 26,8\% (IC95\%: $25,1-28,6)$ para aqueles de 11 ou mais anos de escolaridade. A probabilidade de uma declaração de saúde regular/ruim no grupo de pessoas sem ocupação foi 1,20 vez a da observada no grupo com ocupação. Embora não tenha alcançado significância estatística, os indivíduos sem renda apresentaram risco estimado 1,25 vez a mais para pior percepção de saúde quando comparados àqueles com renda familiar de cinco ou mais salários mínimos.

O índice de insatisfação com a saúde apresentou-se significativamente maior para as pessoas com morbidade autorreferida, indivíduos inativos e com obesidade, respectivamente (Tabela 3).

Metade das pessoas com morbidade autorreferida (50,1\%; IC95\%: 48,6-51,6) relatou ter a saúde regular/ruim. Elas apresentaram razão de prevalência de percepção não boa de saúde 2,29 vezes mais elevada do que aquelas pessoas sem morbidades autorreferidas. Encontrou-se associação entre autoavaliação de estado de saúde com o IMC. A obesidade apresentou-se como categoria de risco, com significância estatística, indicando que os indivíduos obesos têm probabilidade de se autodeclarar com saúde não boa $48 \%$ maior quando comparados àqueles de peso

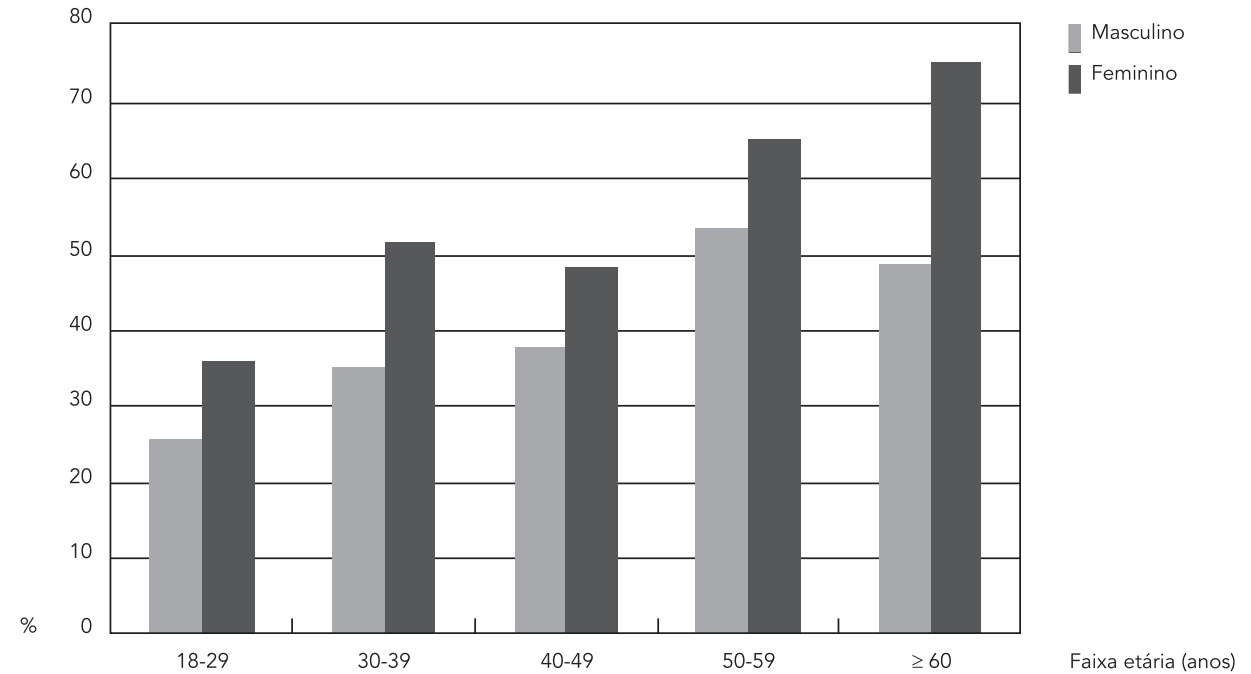


Prevalências e razões de prevalência de percepção "insatisfatória" de saúde em pessoas com 18 anos ou mais, segundo variáveis sociais e demográficas. Rio Branco, Acre, Brasil, 2007-2008 ( $n=1.514)$.

\begin{tabular}{|c|c|c|c|}
\hline Perfil sociodemográfico * & $\begin{array}{c}\text { Percepção "insatisfatória" } \\
\text { de saúde (\%) }\end{array}$ & RP bruta (IC95\%) & RP ajustada ** (IC95\%) \\
\hline \multicolumn{4}{|l|}{ 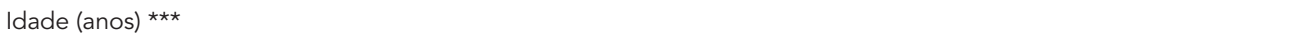 } \\
\hline $18-59$ & $526(40,79)$ & 1,00 & 1,00 \\
\hline$\geq 60$ & $112(62,85)$ & $1,54(1,32-1,79)$ & $1,36(1,19-1,56)$ \\
\hline Sexo \# & & 1,00 & 1,00 \\
\hline Masculino & $231(35,81)$ & $1,39(1,05-1,85)$ & $1,38(1,01-1,88)$ \\
\hline Feminino & $407(50,03)$ & & \\
\hline \multicolumn{4}{|c|}{ Cor de pele/etnia autodeclarada } \\
\hline \multicolumn{4}{|c|}{ negra/parda } \\
\hline Não & $91(35,80)$ & 1,00 & 1,00 \\
\hline Sim & $546(45,24)$ & $1,26(0,99-1,60)$ & $1,27(1,00-1,61)$ \\
\hline \multicolumn{4}{|l|}{ Escolaridade (anos) } \\
\hline 11 ou mais & $69(26,80)$ & 1,00 & 1,00 \\
\hline $8-10$ & $196(35,16)$ & $1,31(1,01-1,69)$ & $1,30(1,01-1,66)$ \\
\hline $1-7$ & $324(53,87)$ & $2,00(1,52-2,65)$ & $1,79(1,39-2,31)$ \\
\hline 0 & $46(56,58)$ & $2,11(1,40-3,17)$ & $1,67(1,17-2,39)$ \\
\hline Valor de $p$ de tendência & & $<0,001$ & $<0,001$ \\
\hline \multicolumn{4}{|l|}{ Estado civil } \\
\hline Com companheiro & $373(48,33)$ & 1,00 & 1,00 \\
\hline Sem companheiro & $265(37,78)$ & $0,78(0,62-0,98)$ & $0,81(0,67-0,97)$ \\
\hline \multicolumn{4}{|l|}{ Com ocupação } \\
\hline $\operatorname{Sim}$ & $308(36,38)$ & 1,00 & 1,00 \\
\hline Não & $330(51,90)$ & $1,40(1,21-1,63)$ & $1,23(1,09-1,38)$ \\
\hline \multicolumn{4}{|c|}{ Renda familiar (salários mínimos) } \\
\hline 5 ou mais & $18(35,46)$ & 1,00 & 1,00 \\
\hline $3<5$ & $66(33,73)$ & $0,95(0,68-1,31)$ & $0,95(0,68-1,32)$ \\
\hline$<1<3$ & $344(45,68)$ & $1,28(0,98-1,68)$ & $1,25(0,98-1,63)$ \\
\hline 0 & $194(44,44)$ & $1,25(0,94-1,65)$ & $1,25(0,91-1,73)$ \\
\hline Valor de $\mathrm{p}$ de tendência & & $<0,001$ & $<0,001$ \\
\hline
\end{tabular}

* As diferenças nos valores absolutos das frequências correspondem às perdas;

** Razão de prevalência ajustada para idade, sexo e declaração de morbidade autorreferida;

*** Razão de prevalência ajustada somente por sexo e declaração de morbidade autorreferida;

\# Razão de prevalência ajustada somente por idade e declaração de morbidade autorreferida.

normal. A estimativa de risco para autopercepção de saúde insatisfatória foi $74 \%$ maior nos indivíduos que não praticam atividade física em comparação com os que praticam, após ajuste para idade, sexo e presença de morbidade autorreferida.

As pessoas que referiram consumo de bebida alcoólica apresentaram menor probabilidade de declarar o estado de saúde como regular/ruim (com significância estatística).

\section{Discussão}

A população do estudo foi caracterizada por predominância das mulheres, com idade mediana de 38,8 anos, de raça/cor autodeclarada parda, com baixas escolaridade e renda mensal, por ter companheiro e exercer atividade laboral. A prevalência de autoavaliação insatisfatória da saúde foi elevada tanto na população adulta (18-59 anos), quanto nos idosos.

O presente estudo apontou diferenças no padrão de percepção do estado de saúde da população de Rio Branco. Esses resultados foram 
Prevalências e razões de prevalência de percepção "insatisfatória" de saúde em pessoas com 18 anos ou mais, segundo variáveis do estilo de vida e de situação de saúde. Rio Branco, Acre, Brasil, 2007-2008.

\begin{tabular}{|c|c|c|c|}
\hline Características de vida/saúde * & $\begin{array}{c}\text { Percepção "insatisfatória" } \\
\text { de saúde (\%) }\end{array}$ & RP bruta (IC95\%) & RP ajustada ** (IC95\%) \\
\hline \multicolumn{4}{|l|}{ Fuma ou já fumou } \\
\hline Não & $332(38,03)$ & 1,00 & 1,00 \\
\hline $\operatorname{Sim}$ & $306(51,67)$ & $1.35(1,13-1,62)$ & $1,22(1,03-1,43)$ \\
\hline \multicolumn{4}{|l|}{ Bebe } \\
\hline Não & $494(48,91)$ & 1,00 & 1,00 \\
\hline Sim & $144(30,92)$ & $0,65(0,53-0,74)$ & $0,74(0,65-0,85)$ \\
\hline \multicolumn{4}{|l|}{ Prática de atividade física } \\
\hline Sim & $86(24,99)$ & 1,00 & 1,00 \\
\hline Não & $332(41,44)$ & $1,93(1,43-2,07)$ & $1,74(1,35-2,24)$ \\
\hline \multicolumn{4}{|l|}{ Hábito alimentar saudável } \\
\hline Sim & $133(42,44)$ & 1,00 & 1,00 \\
\hline Não & $505(44,09)$ & $1,03(0,87-1,23)$ & $1,08(0,92-1,28)$ \\
\hline \multicolumn{4}{|l|}{ Índice de massa corporal } \\
\hline Eutrófico & $262(37,00)$ & 1,00 & 1,00 \\
\hline Baixo peso & $25(47,24)$ & $1,27(0,90-1,79)$ & $1,27(0,89-1,81)$ \\
\hline Sobrepeso & $196(44,67)$ & $1,20(1,05-1,37)$ & $1,11(0,99-1,24)$ \\
\hline Obeso & $147(61,56)$ & $1,66(1,39-1,98)$ & $1,48(1,26-1,73)$ \\
\hline Valor de $\mathrm{p}$ de tendência & & $<0,001$ & $<0,001$ \\
\hline \multicolumn{4}{|l|}{ Morbidade autorreferida } \\
\hline Não & $81(20,48)$ & 1,00 & 1,00 \\
\hline $\operatorname{Sim}$ & $557(50,12)$ & $2,44(1,99-3,00)$ & $2,29(1,86-2,83)$ \\
\hline \multicolumn{4}{|l|}{$\begin{array}{l}\text { Número de morbidades } \\
\text { autorreferidas } \star \star \star\end{array}$} \\
\hline Nenhuma & $81(20,48)$ & 1,00 & 1,00 \\
\hline $1-3$ & $351(41,53)$ & $2,02(1,62-2,52)$ & $1,97(1,58-2,46)$ \\
\hline $4-6$ & $173(73,39)$ & $3,58(2,96-4,33)$ & $3,32(2,73-4,04$ \\
\hline $7-9$ & $30(81,31)$ & $3,97(3,19-4,93)$ & $3,83(3,11-4,72)$ \\
\hline 10 ou + & $3(100)$ & $4,88(4,11-5,79)$ & $4,48(3,23-6,22)$ \\
\hline Valor de $\mathrm{p}$ de tendência & & $<0,001$ & $<0,001$ \\
\hline
\end{tabular}

* As diferenças nos valores absolutos das frequências correspondem às perdas;

** Razão de prevalência ajustada para idade, sexo e declaração de morbidade autorreferida;

*** Variável ajustada somente por idade e sexo.

consistentes com os de outros inquéritos de base populacional referentes à avaliação do estado de saúde realizados no Brasil, mesmo considerando as diferenças metodológicas empregadas nestas pesquisas. Embora a maioria da população estudada tenha declarado satisfação com seu estado de saúde (excelente ou boa), chama a atenção a elevada prevalência do menor nível de avaliação da saúde (ruim). Tal fato torna-se preocupante, uma vez que diversos autores têm constatado o alto poder preditivo de morbidade e mortalidade decorrente da avaliação precária da própria saúde 4,5,6,16,23,24. Destacam-se os da- dos quanto à razão de prevalência de autoavaliação negativa de saúde com inatividade física, fumo, obesidade e a carga de morbidade referida, corroborando os achados recentes de outras investigações 16,17,18,25,26.

Várias investigações 2,23 têm referido que avaliação subjetiva da saúde permite verificar outros parâmetros de saúde para além da condição físico-patológica dos indivíduos. Shooshtari \& Shields 2 referem que as pessoas que não apresentam problemas de saúde específicos normalmente não classificam a sua saúde como sendo "boa" ou "excelente", mas como "regular". Acres- 
centa, ainda, que os indivíduos que fazem uma avaliação da sua saúde como negativa apresentam frequentemente problemas de saúde de ordem física, enquanto as avaliações mais positivas revelam uma preocupação mais abrangente da saúde, no nível de bem-estar geral.

Uma análise do inquérito VIGITEL 13, realizado com 54.339 indivíduos adultos, distribuídos nas capitais dos 26 estados brasileiros e no Distrito Federal em 2010 (sendo pelo menos dois mil em cada capital), verificou que a porcentagem de percepção ruim da saúde da população brasileira oscilou entre 1,5\% (IC95\%: 0,2-2,8) e 11,8\% (IC95\%: 7,2-16,3). Neste estudo, ainda que a prevalência de percepção ruim em Rio Branco $(8,1 \%$; IC95\%: 7,5-8,7) tenha sido um pouco inferior à encontrada pelo VIGITEL, observa-se similaridade entre os dados das duas investigações, evidenciada pela superposição dos intervalos de confiança.

Os dados do inquérito realizado pelo Instituto Nacional de Câncer 13, com 23.442 indivíduos de 15 anos ou mais, distribuídos em 15 capitais brasileiras e mais o Distrito Federal, no período de 2002 a 2003, com amostragem semelhante à do presente estudo, mostraram que a percepção da saúde regular ou ruim variou entre 18,4\% (IC95\%: 15,9-20,8) e 36\% (IC95\%: 32,5-39,4), proporções inferiores às encontradas nesta pesquisa (43,7\%; IC95\%: 39,6-44,6). É importante a ressalva de que participaram daquele inquérito apenas duas capitais da Região Norte, que estão entre as cinco cidades onde foram encontrados os maiores valores de percepção negativa da saúde, ocupando o primeiro (Belém) e o quinto (Manaus) lugares. Dessa forma, parece que os fatores socioeconômicos e culturais peculiares da região Norte favorecem, possivelmente, o estado precário da saúde dessas populações 12 .

Destaca-se, ainda, que avaliação negativa (ruim) da saúde verificada em Rio Branco $(8,1 \%$; IC95\%: 7,5-8,7) chega a ser praticamente o dobro da encontrada globalmente na população brasileira (3,8\%), em 2008, pela PNAD (ruim/muito ruim) 27 . Contudo, esse resultado assemelha-se à porcentagem encontrada na Pesquisa Mundial de Saúde (9\%) 28, com dados de 2003.

Comparando com os dados do National Health Interview Survey (NHIS), observou-se que o perceptual de avaliação de estado de saúde como regular ou ruim da população de Rio Branco é aproximadamente três vezes maior que a relatada pela população dos Estados Unidos (13\%) em 2008. Dados do Joint Canada/United States Survey of Health (JCUSH) demonstraram que, no Canadá, a frequência de saúde regular/ruim (11\%) foi cerca de um quarto da verificada em nosso estudo 23,24.
Na esfera coletiva, os fatores demográficos e socioeconômicos podem contribuir para as variações de autoavaliação de saúde entre os diferentes indivíduos do Município de Rio Branco. Como se pôde perceber, a análise das associações entre fatores sociodemográficos e autoavaliação de saúde mostrou que indivíduos com baixa escolaridade, do sexo feminino em idades mais avançadas, de raça/cor negra ou parda e sem ocupação julgaram mais negativamente seu estado de saúde, o que coincide com os fatores que interferem na autoavaliação de saúde descrita na literatura 4,6,14,15,23,24,29,30.

É possível que a associação direta da posição socioeconômica, dada pela escolaridade, seja fortemente influenciada por contextos sociais e culturais que estão relacionados com a crença sobre saúde, a percepção e a motivação que se tem sobre a realidade, contextos estes que têm valor primordial na determinação do comportamento e do estilo de vida. Verifica-se, portanto, a necessidade de se diminuirem as desigualdades de percepção de saúde por meio do aumento da equidade no acesso ao sistema escolar e na permanência neste 8,17,26,31.

Essa associação indica que a autopercepção é mais rigorosa no que se refere ao nível de satisfação com a própria saúde. Embora, na ótica médica, o "estado de saúde" refira-se a uma anormalidade patológica indicada por um conjunto de sinais e sintomas, a autoavaliação é subjetiva, combinando os componentes físico e emocional, incluindo a sensação de bem-estar e satisfação com a vida 6,32 .

Alguns fatores são identificados como preditores da diferença entre os gêneros na avaliação do estado de saúde. As mulheres têm maior preocupação, conhecimento e compromisso quanto ao cuidado da sua saúde e da família. Além disso, apresentam maior busca e adesão ao tratamento de doenças do que os homens, uma vez que as mudanças comportamentais, de estilo de vida e de saúde pelas quais elas têm passado nos últimos anos podem ter favorecido o aparecimento de doenças crônicas 32,33,34.

Dados do suplemento sobre Acesso e Utilização dos Serviços de Saúde 27, da PNAD 2008, revelam que as mulheres são majoritariamente as que mais utilizam os serviços de saúde no Brasil. A pesquisa, considerando o total da população brasileira, revelou que 73,6\% (139,9 milhões de habitantes) declararam ter um serviço de saúde de uso regular; destes, 53,4\% eram mulheres. Nos 12 meses que antecederam a entrevista, $67,7 \%$ da população brasileira declarou ter consultado um médico. A parcela de mulheres que o fizeram no último ano foi de $76,1 \%$; a de homens, 58,8\%. 
O percentual de autoavaliação da saúde regular/ruim dos idosos da nossa investigação $(62,8 \%)$ foi maior do que o encontrado no estudo Saúde, Bem-estar e Envelhecimento (SABE) 34 , realizado entre os anos 2000 e 2001, com 5.064.907 pessoas de 60 anos ou mais, em sete cidades da América Latina e Caribe. Nesse estudo, a cidade brasileira avaliada, São Paulo, ficou em quarto lugar, ao se considerarem os percentuais de pior autopercepção da saúde $(53,4 \%)$, perdendo apenas para México DF, no México $(69,4 \%)$, Ciudad de La Habana, em Cuba (62,6\%) e Santiago, no Chile (63,2\%).

Estudos recomendam a implementação de ações integradas que colaborem para a redução das desigualdades com consequente melhoria nas condições de vida e de saúde da população. Tal recomendação justifica-se pelo fato de se conhecer o forte poder preditivo de morbimortalidade da pior percepção de saúde, independentemente da identificação médica de doenças 7,9,35.

Dentre as limitações inerentes às investigações de delineamento transversal, destaca-se a possibilidade de esta pesquisa apresentar viés de causalidade reversa, que pode ocorrer quando aparente exposição é consequência do desfecho. Esses aspectos talvez expliquem a inexistência de associação de percepção insatisfatória de saúde para as variáveis hábito alimentar e etilismo, como observado em outros estudos 17,18.

\section{Conclusão}

A percepção de saúde mais prevalente no município de Rio Branco foi: "boa”, seguida por "regular”. O percentual de autoavaliação insatisfatória da saúde (regular/ruim) foi muito elevado, se comparado ao das capitais brasileiras de melhor situação socioeconômica e com dados populacionais de países desenvolvidos. A percepção insatisfatória da saúde foi proporcionalmente maior em indivíduos com baixa instrução, idosos, do sexo feminino, de cor de pele/etnia autodeclarada preta ou parda, com companheiro e sem ocupação. Portanto, os fatores sociais e econômicos mostraram-se relevantes na percepção de saúde da população.

Quanto às variáveis de estilo de vida, as maiores prevalências de percepção negativa da saúde foram observadas entre indivíduos que apresentavam inatividade física e sobrepeso. O maior risco de pior autoavaliação da saúde foi encontrado entre aqueles que declararam ter pelo menos um agravo à saúde.

Diante dos achados deste estudo, tornam-se necessárias medidas de controle e prevenção dos riscos à saúde. Para que sejam implementadas políticas de promoção de vida saudável, é importante conhecer os determinantes desses indicadores de morbidade autorreferida de saúde em Rio Branco.

Sugere-se, ainda, que os resultados desta investigação sejam monitorados por inquéritos de base populacional periódicos, a fim de verificar a determinação das associações observadas, podendo, futuramente, detectar associação com mortalidade nesta população, além de nortear e avaliar estratégias de promoção de saúde. Aponta-se a necessidade, também, de estudos qualitativos para verificar aspectos específicos ao tema não viabilizados por este método. Uma possibilidade seria um estudo quanto ao perfil dissociado entre sexos acerca da concepção de saúde para adicionar outras variáveis que podem influenciar nessa relação às informações quantitativas. 


\section{Resumo}

O conceito subjetivo de saúde incluso no processo de autoavaliação de saúde possibilita identificar os fatores que estão associados à percepção de vida saudável das populações. Objetivou-se verificar a autoavaliação de saúde e os fatores associados em Rio Branco, Acre, Brasil, por meio de um estudo transversal de base populacional com amostra composta de 1.516 adultos, entre 2007-2008. A análise multivariada foi realizada por meio de regressão de Poisson, adotando-se, como variável dependente, a percepção regular/ruim do estado de saúde. Diferenças entre gênero, grupo etário, nível de instrução e renda desfavoráveis afetaram negativamente a percepção de saúde dos indivíduos. Após ajuste para idade, sexo e morbidade autorreferida, os riscos estimados para percepção "não boa" de saúde foram maiores para os inativos, obesos e indivíduos com alguma morbidade autorreferida. Os resultados sugerem que as diferenças de condições de vida dos residentes em Rio Branco estão relacionadas com a autoavaliação precária do estado de saúde.

Nível de Saúde; Diagnóstico da Situação em Saúde; Perfil de Saúde; Inquéritos Epidemiológicos

\section{Referências}

1. Neri AL. Qualidade de vida na velhice e subjetividade. In: Neri AL, organizador. Qualidade de vida na velhice: enfoque multidisciplinar. Campinas: Alínea; 2007. p. 13-59.

2. Shooshtari S, Shields M. Determinants of self-perceived health. Health Rep 2001; 13:35-51.

3. Canguilhem G. O normal e o patológico. 5a Ed. Rio de Janeiro: Forense Universitária; 2000.

4. Franks P, Gold MR, Fiscella K. Sociodemographics, self-rated health, and mortality in the US. Soc Sci Med 2003; 56:2505-14.

5. Vuorisalmi M, Lintonen T, Jylha M. Global self-rated health data from a longitudinal study predicted mortality better than comparative self-rated health in old age. J Clin Epidemiol 2005; 58:680-7.

6. Molarius A, Janson S. Self-rated health, chronic diseases, and symptoms among middle-aged and elderly men and women. J Clin Epidemiol 2002; 55:364-70.

\section{Colaboradores}

P. C. L. Bezerra participou da concepção, processamento, análise e interpretação dos dados, da revisão da literatura e redação do artigo. S. P. Opitz e R. J. Koifman participaram da organização, análise e interpretação dos dados e da revisão final do artigo. P. T. Muniz participou da coleta de dados e revisou a versão final do artigo.

\section{Agradecimentos}

Ao CNPq (Edital Casadinho UFAC-FIOCRUZ, processo $\mathrm{n}^{\circ}$. 620024/2008-9) e CAPES (Programas PROCAD-NF 1442/2007 e PROCAD-NF 2557/2008), pelo apoio ao programa de colaboração entre o Programa de Mestrado em Saúde Coletiva da Universidade Federal do Acre e o Programa de Pós-graduação em Saúde Pública e Meio Ambiente da Fundação Oswaldo Cruz.
7. Mackenbach JP, Kunst AE, Cavelaars AEJM, Gronhof F, Geurts JJM. Socioeconomic inequalities in morbidity and mortality in Western Europe. The EU Working Group on Socioeconomic Inequalities in Health. Lancet 1997; 349:1655-9.

8. Sadana R, Tandon A, Murray CJL, Serdobova S, Cao Y, Xie WJ, et al. Describing population health in six domains: comparable results from 66 household surveys. Geneva: World Health Organization; 2002. (GPE Discussion Paper, 43).

9. Dachs JNW. Determinantes das desigualdades na auto-avaliação do estudo de saúde no Brasil: análise dos dados da PNAD/1998. Ciênc Saúde Coletiva 2002; 7:641-57.

10. Cesar CLG, Carandina L, Alves MCGP, Barros MBA, Goldbaum M, organizadores. Saúde e condição de vida em São Paulo. Inquérito multicêntrico de saúde no Estado de São Paulo - ISA-SP. 1a Ed. São Paulo: Faculdade de Saúde Pública, Universidade de São Paulo; 2005. 
11. Dachs JNW, Santos APR. Auto-avaliação do estado de saúde no Brasil: análise dos dados da PNAD/2003. Ciênc Saúde Coletiva 2006; 11:887-94.

12. Coordenação de Prevenção e Vigilância, Instituto Nacional de Câncer. Inquérito domiciliar sobre comportamentos de risco e morbidade referida de doenças e agravos não transmissíveis: Brasil, 15 capitais e Distrito Federal 2002-2003. Rio de Janeiro: Instituto Nacional de Câncer; 2004.

13. Secretaria de Vigilância em Saúde, Ministério da Saúde. VIGITEL Brasil 2010. Vigilância de fatores de risco e proteção para doenças crônicas por inquérito telefônico. Brasília: Ministério da Saúde; 2011.

14. Szwarcwald CL, Souza-Júnior PRB, Esteves MAP, Damacena GN, Viacava F. Socio-demographic determinants of self-rated health in Brazil. Cad Saúde Pública 2005; 21 Suppl 1:S54-S64.

15. Traebert J, Bortoluzzi MC, Kehrig RT. Auto-percepção das condições de saúde da população adulta, Sul do Brasil. Rev Saúde Pública 2011; 45:789-93.

16. Pereira JC, Barreto SM, Passos VMA. Perfil de risco cardiovascular e autoavaliação da saúde no Brasil: estudo de base populacional. Rev Panam Salud Pública 2009; 25:491-8.

17. Fonseca AS, Blank VLG, Barros MVG, Nahas MV. Percepção de saúde e fatores associados em industriários de Santa Catarina, Brasil. Cad Saúde Pública 2008; 24:567-76.

18. Lino MZR, Muniz PT, Siqueira KS. Prevalência e fatores associados ao excesso de peso em adultos: inquérito populacional em Rio Branco, Acre, Brasil, 2007-2008. Cad Saúde Pública 2011; 27:797810.

19. Secretaria de Vigilância em Saúde, Ministério da Saúde. VIGITEL Brasil 2008. Vigilância de fatores de risco e proteção para doenças crônicas por inquérito telefônico. Brasília: Ministério da Saúde; 2009.

20. World Health Organization. Recommended amount of physical activity. http://www.who.int/ dietphysicalactivity/fatsheet_recommendations/ en (acessado em 16/Out/2009).

21. Figueiredo ICR, Jaime PC, Monteiro CA. Fatores associados ao consumo de frutas, legumes e verduras em adultos da cidade de São Paulo. Rev Saúde Pública 2008; 42:777-85.

22. World Health Organization. Obesity: preventing and managing the global epidemic. Geneva: World Health Organization; 2000. (Technical Report Series, 894).

23. Pleis JR, Lucas JW, Ward BW. Summary health statistics for U.S. adults: National Health Interview Survey, 2008. Vital Health Stat 10 2009; (242):1-157.
24. Duclos JY, Écheviny D. Health and income: a robust comparison of Canada and the US. Montréal: Centre Interuniversitaire sur le Risque, les Politiques Économiques et l'Emploi; 2009. (Cahier de Recherche/Working Paper, 09-09).

25. Theme-Filha MM, Szwarcwald CL, Souza-Júnior PRB. Socio-demographic characteristics, treatment coverage, and self-rated health of individuals who reported six chronic diseases in Brazil, 2003. Cad Saúde Pública 2005; 21 Suppl:S43-53.

26. Theme-Filha M, Szwarcwald MC, Souza Júnior PRB. Medidas de morbidade referida e inter-relações com dimensões de saúde. Rev Saúde Pública 2008; 42:73-81.

27. Instituto Brasileiro de Geografia e Estatística. Pesquisa Nacional por Amostra de Domicílios (PNAD). Síntese de indicadores 2008. Rio de Janeiro: Instituto Brasileiro de Geografia e Estatística; 2009.

28. Szwarcwald CL, Viacava F, Vasconcellos MTL, Leal MC, Azevedo LO, Queiroz RSB, et al. Pesquisa Mundial de Saúde 2003: o Brasil em números. Radis 2004; 23:14-33.

29. Paskulin LMG, Vianna LAC. Perfil sociodemográfico e condições de saúde auto-referidas de idosos de Porto Alegre. Rev. Saúde Pública 2007; 41:75768.

30. Mendoza-Sassi RA, Béria JU. Gender differences in self-reported morbidity: evidence from a population-based study in southern Brazil. Cad Saúde Pública 2007; 23:341-6.

31. Souza MC, Otero UB, Figueiredo VC, Lozana JA. Autopercepção do estado de saúde na área urbana de 18 municípios de capital do Brasil. Rev Bras Epidemiol 2008; 11 Suppl 1:193.

32. Sousa ALL. Educando a pessoa hipertensa. In: Pierin AMG, organizador. Hipertensão arterial: uma proposta para o cuidar. Barueri: Manole; 2004. p. 165-84.

33. Pires CGS, Mussi FC. Crenças em saúde para o controle de hipertensão arterial. Ciênc Saúde Coletiva 2008; 13 Suppl 2:2257-67.

34. Menéndez J, Guevara A, Arcia N, Díaz EML, Marín C, Alfonso JC. Enfermedades crónicas y limitación funcional en adultos mayores: estudio comparativo en siete ciudades de América Latina y el Caribe. Rev Panam Salud Pública 2005; 17:353-61.

35. Werneck GL. Considerações metodológicas à luz da Pesquisa Mundial de Saúde no Brasil, 2003. Rev Bras Epidemiol 2008; 11 Suppl 1:67-71.

Recebido em 30/Mar/2011

Versão final reapresentada em 10/Ago/2011

Aprovado em 22/Ago/2011 